\title{
Band gap engineering in simultaneous phononic and photonic crystal slabs
}

\author{
B. Djafari Rouhani $\cdot$ Y. Pennec $\cdot$ E.H. El Boudouti . \\ J.O. Vasseur · Y. El Hassouani · C. Li • A. Akjouj • \\ D. Bria
}

Received: 13 January 2010 / Accepted: 3 December 2010 / Published online: 29 December 2010

(c) Springer-Verlag 2010

\begin{abstract}
We discuss the simultaneous existence of phononic and photonic band gaps in two types of phononic crystals slabs, namely periodic arrays of nanoholes in a Si membrane and of $\mathrm{Si}$ nanodots on $\mathrm{SiO}_{2}$ membrane. In the former geometry, we investigate in detail both the boron nitride lattice and the square lattice with two atoms per unit cell (these include the square, triangular and honeycomb lattices as particular cases). In the latter geometry, some preliminary results are reported for a square lattice.
\end{abstract}

\section{Introduction}

Phononic crystals $[1,2]$, constituted by a periodical repetition of inclusions in a matrix background, has received a great deal of attention during the last two decades [3]. Associated with the possibility of absolute band gaps in their band structure, these materials have found several potential applications, in particular in the field of wave guiding and filtering (in relation to the properties of their linear and point defects) as well as in the field of sound isolation. Another issue of interest is based on the refractive properties of these materials for exploring phenomena such as negative refraction, focusing, self-collimation and beam splitting as well as

B. Djafari Rouhani $(\bowtie) \cdot$ Y. Pennec $\cdot$ E.H. El Boudouti · J.O. Vasseur · Y. El Hassouani - C. Li · A. Akjouj · D. Bria Institut d'Electronique, de Microélectronique et de Nanotechnologie, UMR CNRS 8520, Université de Lille1

Sciences et Technologies, Cité scientifique, 59652 Villeneuve d'Ascq, France

e-mail: bahram.djafari-rouhani@univ-lille1.fr

E.H. El Boudouti · D. Bria

Laboratoire de Dynamique et d'Optique des Matériaux, Faculté des Sciences, Université d'Oujda, Oujda, Morocco for the realization of metamaterials for controlling the propagation of sound.

The study of slabs of phononic crystals has become a topic of major interest since a few years only. Indeed we and other authors have demonstrated [4-7] that with an appropriate choice of the geometrical and physical parameters these finite thickness structures can also exhibit absolute band gaps. This makes them suitable for similar applications as in the case of 2D phononic crystals with the additional property of confinement in the vertical direction. More recently we have proposed a new type of finite thickness phononic crystal constituted by a periodic array of dots (or beams) on a membrane $[8,9]$. In this paper we briefly recall some of the results about the phononic band structure in the above systems. Then, we mainly focus on a detailed study of simultaneous phononic and photonic band gaps in these materials. Indeed, many papers have investigated separately the existence of photonic $[10,11]$ and phononic band gaps, but relatively few works have been devoted to simultaneous control of phonons and photons [12-15] and most of the papers are dealing with the case of 2D structures [12-14]. The two following sections are, respectively, devoted to the geometries of periodic nanoholes in a $\mathrm{Si}$ membrane and periodic array of $\mathrm{Si}$ nanodots deposited on a $\mathrm{SiO}_{2}$ membrane. Most of the calculations are performed with the Plane Wave Expansion (PWE) method and the good convergence of the results is also checked in some cases by using the Finite Difference Time Domain (FDTD) and Finite Element (FE) methods.

\section{Periodic array of holes in a silicon membrane}

In a previous work $[4,5]$, we demonstrated the existence of absolute phononic band gaps in square and honeycomb lattices of holes in a Si membrane provided the thickness of 
the slab is about half of the lattice period and the filling fraction is sufficiently high. Here we consider the more general cases, shown in Fig. 1, of the square lattice with two atoms per unit cell and of the boron nitride (BN) lattice (which includes triangular and honeycomb lattices) and investigate both the phononic and photonic band gaps. By considering the lattice period $a$ as the unit of length, there are several geometrical parameters involved in the problem, namely the thickness $h$ of the slab, the filling fraction $f$ and the ration $\alpha=r_{1} / r_{2}$ of the radii of the two types of holes in the unit cell. The band structures are calculated for a large variety of these parameters in the useful ranges $(h / a$ from 0.4 to $0.7, f$ from 0.3 to 0.7 , and $\alpha$ from 0 to 1 ). The frequencies are given in the dimensionless units $\Omega=\omega a / 2 \pi c$ where $c$ is the velocity of light in vacuum for electromagnetic waves and the transverse velocity of sound in Si for elastic waves. According to the symmetry of the structure with respect to the middle plane of the slab, the modes can be classified into symmetric (even) and antisymmetric (odd) modes. Let us mention that in the slab geometry, the photonic gaps have to be searched only below the light cone in vacuum; however, these gaps should preferably occur at frequencies $\Omega$ below 0.5 , otherwise they will be restricted to a very small area of the Brillouin zone and are therefore not very interesting.

In the square lattice, the trends are the following. On the phononic side, the limitation comes from the odd modes which only display narrow gaps. Moreover, these gaps occur for high filling fraction $(f>0.6$, i.e. very close holes) and
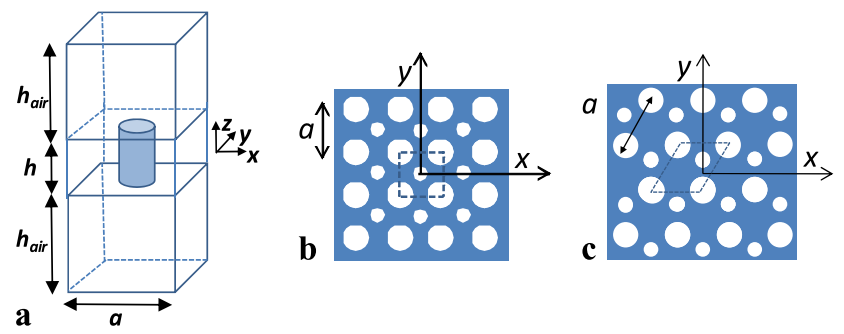

Fig. 1 (a) Representation of the unit cell for the numerical calculations. (b) Square lattice with two atoms per unit cell. (c) Boron Nitride (BN) lattice

Fig. 2 Phononic and photonic band gaps (white areas) in a square lattice with two atoms per unit cell, with $h=0.6 a$ and $f=0.65$. The odd and even photonic modes are shown separately, because they do not overlap. The red arrows represent the domains where there are simultaneous phononic and photonic gaps almost near $\alpha=0$ (simple square) as illustrated in Fig. 2a. On the photonic side, absolute band gaps can only exist for $h / a \sim 0.4$ in a very restricted region of the Brillouin zone (near the M point) just below the light cone; so this solution is not very interesting. At lower frequencies, there is in general no overlap between the gaps of both symmetries. The odd gap mainly occurs near $\alpha=0$ whereas other favorable solutions can be found for even modes when the lattice contains two different atoms in the unit cell (see Figs. 2b and c).

Based on the above discussions, the choice of a crystal can be made by searching a structure that exhibits an absolute phononic band gap though a photonic gap of a given symmetry only. Figure 2 illustrates the evolution of the band gaps for a thickness $h=0.6 a$ of the plate and a filling factor $f=0.65$. The existence of an absolute phononic gap together with a photonic gap of a given symmetry requires $\alpha \leq 0.2$.

In the BN lattice, the following trends are obtained. On the phononic side, the largest gaps are obtained towards the honeycomb lattice $(\alpha=1)$ and then the odd gaps are in general included in the even gaps. However, for $h / a \sim 0.6$ to 0.7 , an odd gap can appear for all BN lattices (from $\alpha=0$ (triangular) to $\alpha=1$ (honeycomb)) whereas the even gaps remain open towards the honeycomb lattice. On the photonic side, we have found a narrow absolute gap only for
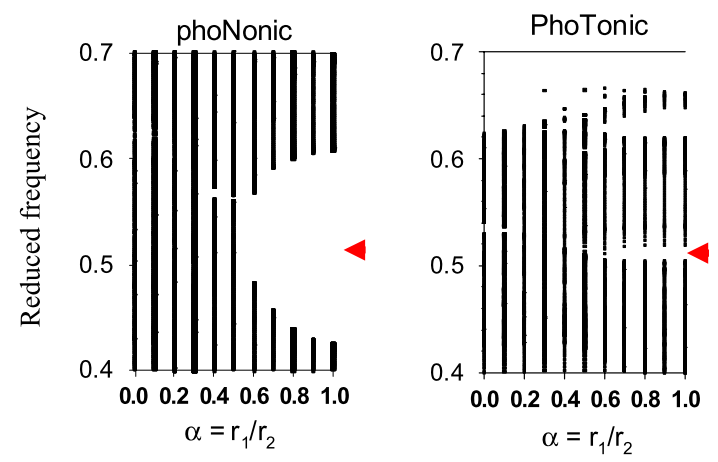

Fig. 3 Absolute phononic and photonic band gaps in a BN lattice with $h=0.4 a$ and $f=0.55$. The frequencies are given in dimensionless units $\Omega=\omega a / 2 \pi c$
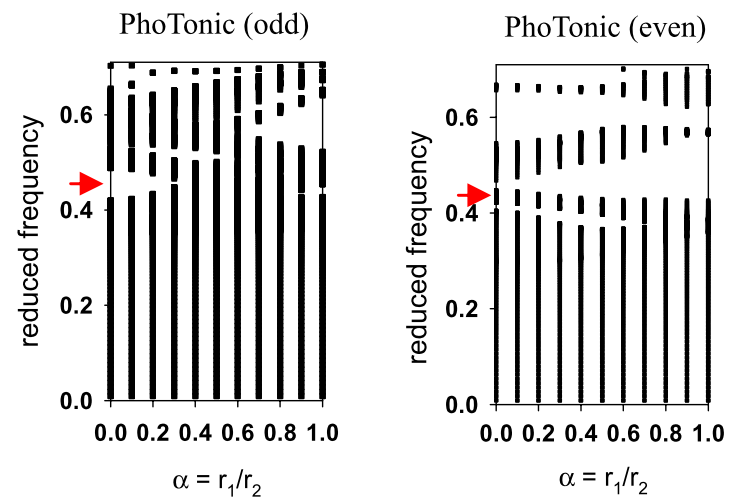

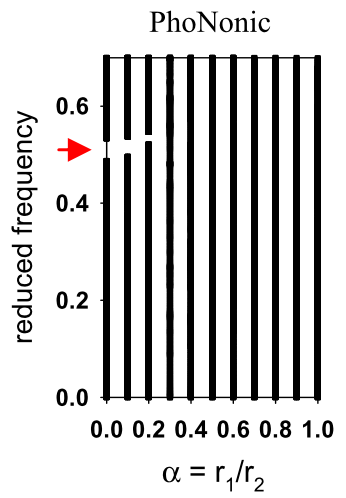


Fig. 4 Phononic and photonic band gaps in a BN lattice with $h=0.6 a, f=0.45$. The odd and even photonic modes are shown separately. The hatched areas represent the domain of $\alpha$ where there are photonic gaps of both symmetries, although at different frequencies

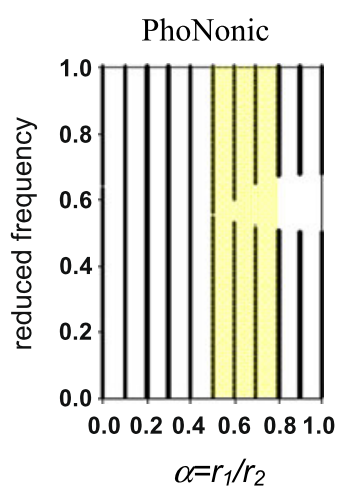

a very restricted range of geometrical parameters, as shown in Fig. 3 for $h / a=0.4$ and $f=0.55$. Otherwise, one can find separate band gaps of even and odd symmetries. The largest gaps of even (resp. odd) symmetry occur towards the triangular (resp. honeycomb) lattice at frequencies around or below (resp. above) $\Omega=0.4$. Nevertheless, the even modes can also display a narrow gap towards the honeycomb lattice provided the thickness of the slab is relatively small $(h / a \sim 0.4-0.5)$ whereas the odd modes can have a narrow gap towards the triangular lattice for large thicknesses $(h / a \sim 0.6-0.7)$.

Again, the choice of a crystal can be made by searching a structure that exhibits an absolute phononic band gap though a photonic gap of a given symmetry only. With this limitation, many possibilities exist in the frame of $\mathrm{BN}$ lattices as illustrated in Fig. 4 for $h / a=0.6$ and $f=0.45$.

More precisely, the following discussion gives the limits of the geometrical parameters for the simultaneous existence of even and odd photonic gaps at two different frequencies. First, the existence of the absolute phononic gap requires a filling factor $f \geq 0.4$ and $\alpha \geq 0.5$. In the photonic side, the thickness of the slab should be taken above $h=0.5 a$ in order to keep the gap in the frequency range below $\Omega=0.5$ (otherwise the gap occurs only in a very restricted range of the Brillouin zone close to the light cone). Finally, a sufficiently wide gap of even symmetry requires $\alpha \leq 0.8$.

Table 1 summarizes the main structures suitable to exhibit an absolute phononic band gap together with photonic band gaps either for both or for only one type of symmetry. Of course, the choice of the lengths for the practical realization of the structure depends on the frequency range of interest for specific applications. For instance, in telecommunication applications, the wavelength of the light is around $1550 \mathrm{~nm}$ in vacuum. Referring to the example mentioned in the last row in Table 1, one obtains the following dimensions of the crystal:

(i) for an even photonic gap, $a=491 \mathrm{~nm}, h=295 \mathrm{~nm}$, $r_{1}=89 \mathrm{~nm}, r_{2}=148 \mathrm{~nm}$, central phononic frequency $=6.7 \mathrm{GHz}$;
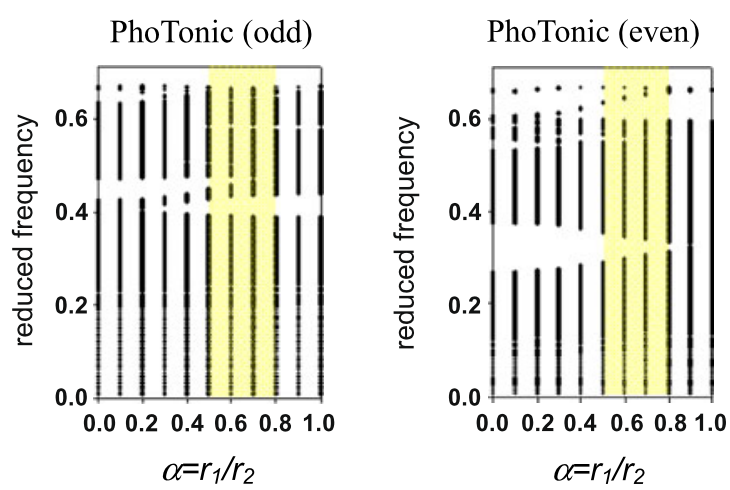

(ii) for an odd photonic gap, $a=637 \mathrm{~nm}, h=382 \mathrm{~nm}, r_{1}=$ $115 \mathrm{~nm}, r_{2}=192 \mathrm{~nm}$, central phononic frequency $=$ $5.1 \mathrm{GHz}$.

\section{Periodic array of dots on a membrane}

In two recent papers [8, 9], we studied the phononic band structure and wave guiding in a new type of phononic crystal constituted by a periodic array of dots deposited on a thin homogeneous membrane (Fig. 5a). Up to now only the square lattice with one atom per unit cell has been investigated. An illustration corresponding to nanodots of $\mathrm{Si}$ on a $\mathrm{SiO}_{2}$ membrane is shown in Fig. $5 b$ with the parameters $h / a=0.8, e / a=0.1$, and $f=0.4$ ( $h$ and $e$ are, respectively, the height of the dots and the thickness of the membrane). A new finding of this work was the possibility of a low-frequency acoustic gap where the wavelengths in the constituting materials are much larger than the typical lengths in the structure (like in the so-called locally resonant materials). The existence of this gap is closely related to the choice of the geometrical parameters which affect the behavior of the lowest dispersion curves and, in particular, the bending of the first three acoustic branches (actually this gap is almost closed in the example of Fig. 5b). Besides, the band structure contains also one or more higher gaps whose number and width are dependent upon the height of the cylinders. So, in this structure, there is in general not too much constraint on the existence of phononic absolute band gaps.

We present now some preliminary results about the existence of photonic gaps. Since we have here one more geometrical parameter than in Sect. 2 (namely the height of the dots), we limit ourselves only to the case of a square lattice. In Fig. 5c, we show that the same structure as before displays an absolute, although relatively narrow, photonic gap. Figure 5d shows the evolution of this gap as a function of the height of the dots for $f=0.4$ : the width of the gap increases when decreasing $h$, but its central frequency increases above 
Table 1 Summary of the most suitable phoxonic crystals and the corresponding band gaps frequencies

\begin{tabular}{|c|c|c|c|c|c|c|c|c|}
\hline Array & $\alpha$ & $f$ & $r / a$ & $h / a$ & $\begin{array}{l}\text { PhoNonic } \\
\text { band gap }\end{array}$ & $\begin{array}{l}\text { PhoTonic } \\
\text { band gap } \\
\text { (odd modes) }\end{array}$ & $\begin{array}{l}\text { PhoTonic } \\
\text { band gap } \\
\text { (even modes) }\end{array}$ & Observations \\
\hline Square & 0 & 0.7 & 0.472 & 0.4 & {$[0.439,0.544]$} & \multicolumn{2}{|c|}{$[0.553,0.658]$} & $\begin{array}{l}\text { Absolute photonic gap in the } \\
\text { neighborhood of } \mathrm{M}\end{array}$ \\
\hline Square & 0 & 0.65 & 0.455 & 0.6 & {$[0.472,0.534]$} & {$[0.410,0.495]$} & {$[0.361,0.400]$} & $\begin{array}{l}\text { High value of } f \text { (holes very close } \\
\text { to each other) }\end{array}$ \\
\hline \multirow[t]{2}{*}{ Honeycomb } & 1 & 0.55 & 0.275 & 0.4 & {$[0.415,0.617]$} & \multicolumn{2}{|c|}{$[0.502,0.531]$} & Small absolute photonic gap \\
\hline & 1 & 0.45 & 0.249 & 0.48 & {$[0.525,0.626]$} & \multicolumn{2}{|c|}{$[0.434,0.454]$} & \\
\hline \multirow[t]{2}{*}{ Honeycomb } & 1 & 0.45 & 0.249 & 0.7 & {$[0.468,0.611]$} & {$[0.368,0.410]$} & I & $\begin{array}{l}\text { Photonic gap of only one } \\
\text { symmetry }\end{array}$ \\
\hline & 1 & 0.45 & 0.249 & 0.4 & {$[0.503,0.588]$} & I & {$[0.445,0.492]$} & \\
\hline \multirow[t]{2}{*}{ Honeycomb } & 1 & 0.6 & 0.289 & 0.7 & {$[0.215,0.605]$} & {$[0.392,0.482]$} & {$[0.474,0.508]$} & $\begin{array}{l}\text { High value of } f \text { (holes very close } \\
\text { to each other) }\end{array}$ \\
\hline & & & & & {$[0.616,0.776]$} & & & \\
\hline \multirow[t]{2}{*}{$\overline{\mathrm{BN}}$} & $\geq 0.5$ & $\geq 0.4$ & & $\geq 0.5$ & & & & Most suitable phoxonic crystals \\
\hline & $\leq 0.8$ & & & & & & & \\
\hline \multirow[t]{2}{*}{ Example } & 0.6 & 0.45 & $r_{1}=0.181$ & 0.6 & {$[0.521,0.602]$} & {$[0.390,0.432]$} & {$[0.291,0.343]$} & \\
\hline & & & $r_{2}=0.302$ & & & & & \\
\hline
\end{tabular}

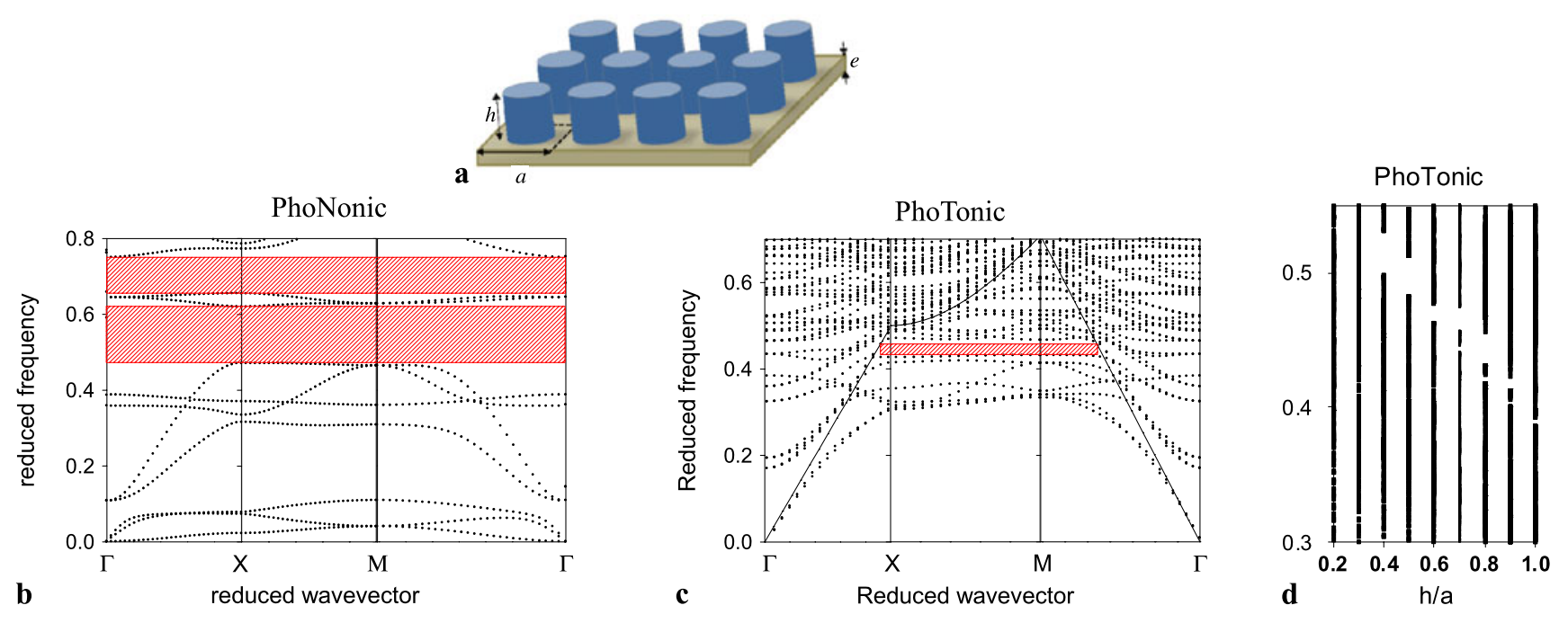

Fig. 5 (a) Schematic of the phononic crystal made up of cylindrical $\mathrm{Si}$ dots on a $\mathrm{SiO}_{2}$ membrane. $h, e$ and $f$ represent, respectively, the height of the dots, the thickness of the plate and the filling factor. (b)

Phononic and (c) photonic dispersions curves calculated for the set of parameters $e=0.1 a, h=0.8 a$ and $f=0.4$. (d) Evolution of the photonic band gap as a function of the height of the dots

0.5 which means that the gap appears then only in a small region of the Brillouin zone around the M point. Now, by decreasing the filling fraction $f$ from 0.4 to 0.3 and then to 0.2 , the gap still persists but go to frequencies above 0.5. On the other hand, by increasing $f$ to 0.5 , the narrow gap closes except when the height of the cylinders is about $h / a \sim 0.8-$ 0.9 . Finally, the photonic gap closes when going to higher filling fraction $f$ (up to 0.7 in our calculation).

\section{Conclusions}

We have shown that BN lattices of holes in a Si membrane can exhibit a complete phononic gap together with either a narrow complete photonic gap or wider photonic gaps of a given symmetry (odd or even) only. Also a structure constituted by a square lattice of dots on a membrane exhibits large complete phononic gaps together with a narrow photonic gap. Other lattices will be investigated in subsequent 
works as well as the properties of linear and point defects in such phoxonic crystals.

Acknowledgement This work is supported in part by the European Commission Seventh Framework programs (FP7) under the FET-Open project TAILPHOX N ${ }^{\circ} 233833$.

\section{References}

1. M.S. Kushwaha, P. Halevi, L. Dobrzynski, B. Djafari-Rouhani, Acoustic band structure of periodic elastic composites. Phys. Rev. Lett. 71, 2022-2025 (1993)

2. M.M. Sigalas, E.N. Economou, Band structure of elastic waves in two dimensional systems. Solid State Commun. 86, 141 (1993)

3. For a comprehensive list of references on phononic crystals, see the phononic database at http://www.phys.uoa.gr/ phononics/phononicDatabase.html

4. J.O. Vasseur, P.A. Deymier, B. Djafari-Rouhani, Y. Pennec, Absolute band gaps in two-dimensional phononic crystal plates, in Proceedings of ASME 2006 International Mechanical Engineering Congress and Exposition (IMECE2006), Chicago, USA, November 2006 (2006), pp. 125-133

5. J.O. Vasseur, P.A. Deymier, B. Djafari-Rouhani, Y. Pennec, A.C. Hladky-Hennion, Absolute forbidden bands and waveguiding in two-dimensional phononic crystal plates. Phys. Rev. B 77, 085415 (2008)

6. J.C. Hsu, T.T. Wu, Efficient formulation for band structure calculations of two-dimensional phononic crystal plates. Phys. Rev. B 74, 144303 (2006)
7. A. Khelif, B. Aoubiza, S. Mohammadi, A. Adibi, V. Laude, Complete band gaps in two-dimensional phononic crystal slab. Phys. Rev. E 74, 046610 (2006)

8. Y. Pennec, B. Djafari-Rouhani, H. Larabi, J.O. Vasseur, A.C. Hladky-Hennion, Low-frequency gaps in a phononic crystal constituted of cylindrical dots deposited on a thin homogeneous plate. Phys. Rev. B 78, 104105 (2008)

9. Y. Pennec, B. Djafari-Rouhani, H. Larabi, A. Akjouj, J.N. Gillet, J.O. Vasseur, G. Thabet, Phonon transport and waveguiding in a phononic crystal made up of cylindrical dots on a thin homogeneous plate. Phys. Rev. B 80, 144302 (2008)

10. S.G. Johnson, S. Fan, P.R. Villeneuve, J.D. Joannopoulos, L.A. Kolodziejski, Guided modes in phononic crystal slabs. Phys. Rev. B 60, 5751-5758 (1999)

11. S. Shi, C. Chen, D.W. Prather, Plane-wave expansion method for calculating bandstructure of phononic crystal slabs with perfectly matched layers. J. Opt. Soc. Am. 21, 1769-1775 (2004)

12. M. Maldovan, E.L. Thomas, Simultaneous localization of photons and phonons in two-dimensional periodic structures. Appl. Phys. Lett. 88, 251907 (2006)

13. M. Maldovan, E.L. Thomas, Simultaneous complete elastic and electromagnetic band gaps in periodic structures. Appl. Phys. B 83, 595-600 (2006)

14. S. Sadat-Saleh, S. Benchabane, F.I. Baida, M. Bernal, V. Laude, Tailoring simultaneous photonic and phononic band gaps. J. Appl. Phys. 106, 074912 (2009)

15. S. Mohammadi, A.A. Eftekhar, A. Adibi, Large simultaneous band gaps for photonic and phononic slabs. OSA/CLEO/QELS (2008) 